\title{
Beneficial effects of the active principle component of Korean cabbage kimchi via increasing nitric oxide production and suppressing inflammation in the aorta of apoE knockout mice
}

\author{
Jeong Sook Noh ${ }^{1}$, Yung Hyun Choi $^{2}$ and Yeong Ok Song ${ }^{1 *}$ \\ ${ }^{1}$ Department of Food Science and Nutrition, Kimchi Research Institute, Pusan National University, Busan 609-735, \\ Republic of Korea \\ ${ }^{2}$ Department of Biochemistry, College of Oriental Medicine, Dong-Eui University and Research Center for Oriental Medicine, \\ Busan 614-052, Republic of Korea
}

(Submitted 12 September 2011 - Final revision received 27 January 2012 - Accepted 27 January 2012 - First published online 13 April 2012)

\section{Abstract}

The present study investigated the effects of $3^{\prime}$-( $4^{\prime}$-hydroxyl- $3^{\prime}, 5^{\prime}$-dimethoxyphenyl)propionic acid (HDMPPA), the active principle compound of kimchi, on vascular damage in the experimental atherosclerotic animal. HDMPPA was administrated by an intraperitoneal injection of $10 \mathrm{mg} / \mathrm{kg}$ per $\mathrm{d}$ for 8 weeks to apoE knockout (KO) mice with an atherogenic diet containing $1 \%$ cholesterol, and its effects were compared with vehicle-treated control mice. HDMPPA increased NO content in the aorta, accompanied by a decrease in reactive oxygen species (ROS) concentration. Furthermore, in the HDMPPA-treated group, aortic endothelial NO synthase (eNOS) expression was up-regulated compared with the control group. These results suggested that HDMPPA could maintain NO bioavailability through an increasing eNOS expression and preventing NO degradation by ROS. Furthermore, HDMPPA treatment in apoE KO mice inhibited eNOS uncoupling through an increase in vascular tetrahydrobiopterin content and a decrease in serum asymmetric dimethylarginine levels. Moreover, HDMPPA ameliorates inflammatory-related protein expression in the aorta of apoE KO mice. Therefore, the present study suggests that HDMPPA, the active compound of kimchi, a Korean functional food, may exert its vascular protective effect through the preservation of NO bioavailability and suppression of the inflammatory response.

Key words: $3^{\prime}$-(4'-Hydroxyl-3' ${ }^{\prime} 5^{\prime}$-dimethoxyphenyl)propionic acid: ApoE knockout mice: Nitric oxide: Endothelial nitric oxide synthase: Inflammation

The progress of atherosclerosis is implicated with the loss of NO bioavailability and increased reactive oxygen species (ROS). In vascular tissue, $\mathrm{NO}$ is derived from L-arginine oxidation by endothelial NO synthase (eNOS), and has a central role in cyclic GMP-mediated vasorelaxation. It has been suggested that NO possesses multiple anti-atherosclerotic properties, which include anti-platelet, anti-proliferative and anti-inflammatory effects ${ }^{(1,2)}$. In atherosclerotic lesions, oxidative stress could be augmented by eNOS uncoupling, which generates superoxide rather than NO. This dysfunction of eNOS activity is caused by an oxidative loss of cofactor, tetrahydrobiopterin $\left(\mathrm{BH}_{4}\right)$ and an increased production of inhibitor, asymmetric dimethylarginine $(\mathrm{ADMA})^{(3-5)}$. Because $\mathrm{NO}$ has been demonstrated as a critical effector molecule in the maintenance of vascular function ${ }^{(6)}$, it is therefore important to maintain NO bioavailability through prevention of eNOS uncoupling during vascular disease.

Vascular inflammation has been suggested to be an important risk factor in the initiation and development of atherosclerosis $^{(7)}$. Inflammatory response in the endothelium promotes leucocyte adhesion and increases vascular permeability via up-regulation of surface cell adhesion molecules and the release of inflammatory cytokines. Endothelial adhesion molecules such as vascular cellular adhesion molecule-1 (VCAM-1) and intercellular adhesion molecule-1 (ICAM-1) are indicative of inflammatory processes. It has been shown that the signal transduction pathways for the expression of adhesion molecules include the activation and translocation of the redox-sensitive transcriptional factor $\mathrm{NF}-\kappa \mathrm{B}^{(8)}$. Additionally, increased ROS and cytokines have

\footnotetext{
Abbreviations: AD, atherogenic diet; ADMA, asymmetric dimethylarginine; $\mathrm{BH}_{4}$, tetrahydrobiopterin; COX-2, cyclo-oxygenase-2; eNOS, endothelial nitric oxide synthase; HDMPPA, $3^{\prime}$-( $4^{\prime}$-hydroxyl- $3^{\prime}, 5^{\prime}$-dimethoxyphenyl)propionic acid; ICAM-1, intercellular adhesion molecule-1; iNOS, inducible nitric oxide synthase; KO, knockout; ROS, reactive oxygen species; VCAM-1, vascular cellular adhesion molecule-1.
} 
been implicated as key mediators of cell signalling pathways that activate and translocate NF- $\mathrm{BB}$ to the nucleus, which in turn up-regulates inflammatory gene expression and aggravates further inflammatory response.

Kimchi, a traditional type of Korean fermented vegetable food, was named in the list of top five 'World Health Foods' for being abundant in dietary fibre, vitamin C, lactic acid bacteria, minerals and other compounds beneficial to health ${ }^{(9)}$. Several scientific trials to identify the potential health benefits of kimchi have been carried out. In our previous studies, plasma cholesterol-lowering effects of kimchi were demonstrated in human subjects ${ }^{(10-12)}$ and animals ${ }^{(13-15)}$, as well as an anti-atherogenic effect having been demonstrated in rabbits ${ }^{(16)}$. From Korean cabbage kimchi, $3^{\prime}-\left(4^{\prime}\right.$-hydroxyl- $3^{\prime}, 5^{\prime}$ dimethoxyphenyl)propionic acid (HDMPPA) with a molecular weight of 226 was isolated and identified as an active principle responsible for inhibiting LDL oxidation and 2,2-diphenyl-1picrylhydrazyl scavenging activity ${ }^{(17)}$. The amount of HDMPPA is approximately $1 \mathrm{mg} / 100 \mathrm{~g} \mathrm{kimchi}{ }^{(15)}$. Next, we chemically synthesised HDMPPA which was biologically identical to the HDMPPA isolated from Korean cabbage kimchi ${ }^{(17)}$. The synthesised HDMPPA showed health benefits on atherosclerosis in rabbits ${ }^{(16)}$. Furthermore, HDMPPA retarded atherosclerotic lesions and ameliorated oxidative stress through inhibiting NADPH oxidase activity in the aorta of apoE knockout $(\mathrm{KO})$ mice $^{(18)}$. However, the effect of HDMPPA on NO metabolism and inflammatory response in the aorta of the atherosclerotic animal model was not determined. In the present study, we investigated the protective effect of HDMPPA on experimental atherosclerosis through the preservation of NO bioavailability and inhibition of the inflammatory response.

\section{Materials and methods}

\section{Materials and reagents}

Chemically synthesised HDMPPA that is biologically identical to the component isolated from cabbage kimchi was used $^{(17)}$. Chemical synthesis was carried out at the Department of Chemistry, Pusan National University, Busan, Republic of Korea. Commercially available kits for plasma TAG and cholesterol analysis were used. The primary antibodies cyclo-oxygenase-2 (COX-2) (sc-19999), inducible NO synthase (iNOS) (sc-7271), eNOS (sc-654), VCAM-1 (sc1504), ICAM-1 (sc-1511), Iк-B $\alpha$ (sc-371), NF-кB p65 (sc-109), $\beta$-actin ( $\mathrm{sc}-47778$ ) and the secondary antibodies of antimouse (sc-2005), anti-rabbit (sc-2004) and anti-goat (sc2020) were purchased from Santa Cruz Biotechnology. Enzymes and other chemicals were purchased from Sigma.

\section{Animals and diets}

For the present study, 6-week-old male apoE KO mice were purchased from Central Lab Animal, Inc. and were randomly divided into two dietary groups: atherogenic diet (AD) only (control, $n$ 15) or AD with HDMPPA treatment (HDMPPA, $10 \mathrm{mg} / \mathrm{kg}$ of body weight, $n$ 15). Each mouse was housed in a room with controlled temperature and lighting and fed an
$\mathrm{AD}$ for 8 weeks. The AD for apoE KO mice was prepared based on the American Institute of Nutrition (AIN)-76 diet by adding $1 \%$ cholesterol and $10 \%$ lard to induce atherosclerosis $^{(19)}$. The diet compositions were as follows (w/w): casein, 20\%; sucrose, 44\%; maize starch, 15\%; cellulose, 5\%; lard, 10\%; AIN-76 mineral mixture, 3.5\%; AIN-76 vitamin mixture, $1 \%$; DL-methionine, $0.3 \%$; choline bitartrate, $0.2 \%$; and cholesterol, 1\%. HDMPPA dissolved in PBS or PBS (as vehicle) was administered every other day by intraperitoneal injection. The dosage for HDMPPA injected into the mice (10 $\mathrm{mg} / \mathrm{kg}$ of body weight per d) was calculated based on results from a previous study that demonstrated anti-atherogenic effects of HDMPPA $(0.33 \mathrm{mg} / \mathrm{kg}$ of body weight per $\mathrm{d})$ in rabbits when it was administered via intravenous injection ${ }^{(16)}$. PBS (vehicle) was injected into the control animals. Mice had free access to food and water. After an 8-week experimental period, the mice were anaesthetised with diethyl ether after $12 \mathrm{~h}$ of fasting. Blood samples were drawn from the inferior vena cava, and plasma was collected immediately after centrifuging ( $800 \boldsymbol{g}$ for $10 \mathrm{~min}$ ). The heart and descending aorta were removed. Of the fifteen aortas, five of them were used for the measurement of ROS and NO, another five for aortic $\mathrm{BH}_{4}$ determination and the remainder for the protein expression study. All samples were stored at $-70^{\circ} \mathrm{C}$ until further analysis. The animal protocol used in this study was reviewed by the Pusan National University Institutional Animal Care and Use Committee on their procedures and scientific care, and the present study was approved (approval no. PNU-2007-00 031).

\section{Nitric oxide concentration of the aorta}

The production of NO in the aorta was measured using cellpermeable diaminofluorescein-2 diacetate (Calbiochem). The aorta was homogenised on ice with $1 \mathrm{~mm}-\mathrm{EDTA}-50 \mathrm{~mm}-$ sodium phosphate buffer ( $\mathrm{pH} 7.4$ ), and then $12.5 \mu \mathrm{M}$-diaminofluorescein-2 diacetate were added to the homogenate. During the reaction time for $30 \mathrm{~min}$, changes in fluorescence were determined at an excitation wavelength of $485 \mathrm{~nm}$ and emission wavelength of $535 \mathrm{~nm}^{(20)}$.

\section{Level of total reactive oxygen species}

Total ROS concentration was measured by the method of Ali et $a{ }^{(21)}$. In brief, the aorta was homogenised on ice with 1 mm-EDTA-50 mm-sodium phosphate buffer ( $\mathrm{pH} 7 \cdot 4)$, and then $25 \mathrm{~mm}-2^{\prime}, 7^{\prime}$-dichlorofluorescein-diacetate were added to the homogenate, and changes in fluorescence for $30 \mathrm{~min}$ were determined at an excitation wavelength of $486 \mathrm{~nm}$ and emission wavelength of $530 \mathrm{~nm}$.

\section{Western blotting analysis}

Aortic tissues were homogenised with ice-cold lysis buffer containing $5 \mathrm{~mm}$-Tris- $\mathrm{HCl}$ ( $\mathrm{pH} 7 \cdot 5$ ), $2 \mathrm{~mm}_{-} \mathrm{MgCl}_{2}, 15 \mathrm{~mm}-$ $\mathrm{CaCl}_{2}$ and $1.5 \mathrm{M}$-sucrose, and then $0 \cdot 1 \mathrm{M}$-dithiothreitol and protease inhibitor cocktail were added. After centrifugation ( $10500 \mathrm{~g}$ for $20 \mathrm{~min}$ at $4^{\circ} \mathrm{C}$ ), the supernatant was used as

a room with controlled temperature and lighting and fed an 


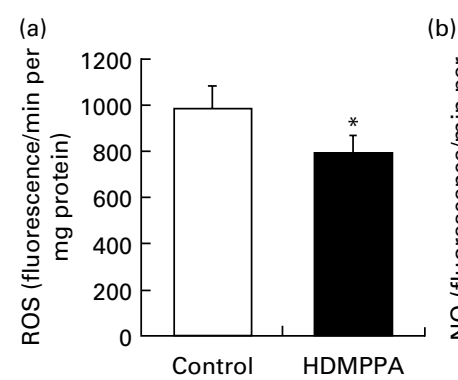

(b)

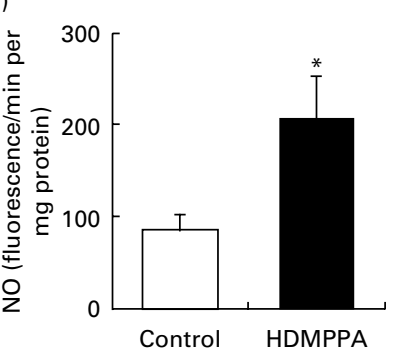

(c)

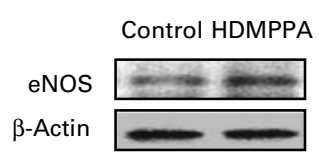

(d)

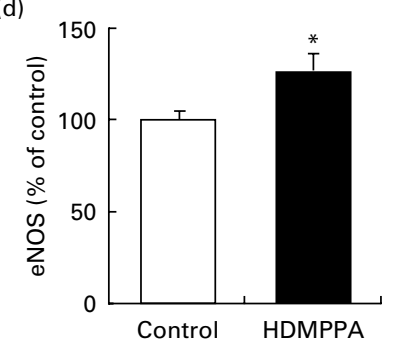

Fig. 1. Effect of $3^{\prime}$-(4'-hydroxyl-3', $5^{\prime}$-dimethoxyphenyl)propionic acid (HDMPPA) on (a) reactive oxygen species (ROS) and (b) nitric oxide (NO) generation, and (c, d) endothelial NO synthase (eNOS) protein expression in the aorta of apoE knockout (KO) mice. Control, PBS (vehicle)-treated apoE KO mice; HDMPPA, HDMPPA $10 \mathrm{mg} / \mathrm{kg}$ of body weight-treated apoE KO mice. Values are means, with their standard errors represented by vertical bars $(n 5) .{ }^{*}$ Mean values were significantly different from those of the control group $(P<0.05)$.

the Western blotting sample. Protein samples $(30 \mu \mathrm{g})$ were analysed by SDS-PAGE in $8 \%$ acrylamide gels for the detection of adhesion molecules and in $10 \%$ gels for the detection of inflammation-related protein (NF-кB p 65 , Iк-B $\alpha$, iNOS, COX-2), and the proteins were then transferred onto nitrocellulose membrane (Whatman $\mathrm{GmbH}$ ). Non-specific binding was blocked by immersing the membrane in 5\% skimmed milk. The membrane was incubated with first antibodies and preserved overnight at $4^{\circ} \mathrm{C}$. After washing with PBS containing $0 \cdot 1 \%$ Tween 20 three times for $40 \mathrm{~min}$, the membrane was further incubated with horseradish peroxidase-conjugated rabbit anti-goat IgG (Santa Cruz Biotechnology) at a 1:5000 dilution for $1 \mathrm{~h}$ at room temperature and detected by enhanced chemiluminescence (Pierce, Life Science).

\section{Detection of tetrahydrobiopterin and total biopterin in the aorta}

Freshly isolated whole aortas were homogenised in extraction buffer containing $50 \mathrm{~mm}$-Tris ( $\mathrm{pH} 7 \cdot 4$ ), $1 \mathrm{~mm}$-dithiotheritol and $1 \mathrm{~mm}$-EDTA at $4^{\circ} \mathrm{C}$, and were centrifuged at $12000 \mathrm{rpm}$ for $15 \mathrm{~min}$ at $4^{\circ} \mathrm{C}^{(22)}$. One whole aorta is needed for $n 1$ experiment. Samples were oxidised under either acidic conditions (with $0.2 \mathrm{M}-\mathrm{HCl}$ containing $50 \mathrm{~mm}-\mathrm{I}_{2}$ ) or alkaline conditions (with $0 \cdot 2 \mathrm{M}-\mathrm{NaOH}$ containing $50 \mathrm{~mm}-\mathrm{I}_{2}$ ). Biopterin content was assessed using HPLC (Agilent 110 Series, Agilent Technologies) with fluorescence detection $(350 \mathrm{~nm}$ excitation, $450 \mathrm{~nm}$ emission). $\mathrm{BH}_{4}$ concentration was calculated as $\mathrm{pmol} / \mathrm{mg}$ protein by subtracting the biopterin peak resulting from alkaline oxidation (accounting for $\mathrm{BH}_{2}$ ) from the biopterin peak resulting from acidic oxidation (accounting for both $\mathrm{BH}_{2}$ and $\mathrm{BH}_{4}$ ).

\section{Detection of asymmetric dimethylarginine in plasma}

The proteins in the conditioned medium were removed using 5-sulfosalicylic acid. The levels of ADMA were measured by HPLC with some modifications ${ }^{(23)}$. O-Phthaldialdehyde adducts of methylated amino acids and internal standard ADMA were monitored using fluorescence detector which was set the excitation wavelengths of $360 \mathrm{~nm}$ and emission of $440 \mathrm{~nm}$ on a Gemini C18 column (Agilent 1100 Series, Agilent Technologies). Samples were eluted from the column using a linear gradient containing mobile phase A composed of $0.05 \mathrm{M}$ ( $\mathrm{pH} 6.8$ ) sodium acetate-methanol-tetrahydrofuran (81:18:1, by vol.) and mobile phase B composed of $0.05 \mathrm{~mm}$ sodium acetate-methanol-tetrahydrofuran (22:77:1, by vol.) at a flow-rate of $1 \mathrm{ml} / \mathrm{min}$.

\section{Statistical analysis}

All data are presented as means with their standard errors. Student's $t$ test was performed to determine statistical significance, with $P<0.05$ considered statistically significant.

\section{Results}

\section{3'-(4'-Hydroxyl-3', 5'-dimethoxyphenyl)propionic acid} prevented the altered nitric oxide bioavailability through inhibition of endothelial nitric oxide synthase uncoupling in the aorta

The induction of eNOS uncoupling generates superoxide rather than $\mathrm{NO}$, consequently enhancing oxidative stress in the atherosclerotic aorta. First, to evaluate the effect of HDMPPA on oxidative stress in the aortas of apoE KO mice, ROS was determined using a fluoro-spectrophotometer (Fig. 1(a)). ROS production was significantly decreased in the aorta treated with HDMPPA ( 788.7 fluorescence/min per mg protein), compared with control (980.1 fluorescence/min per mg protein). Next, NO concentration in the aorta of apoE KO mice was significantly augmented by HDMPPA treatment $(P<0 \cdot 05$, Fig. 1(b)). Finally, HDMPPA treatment elevated the protein level of eNOS in the aorta of apoE KO mice
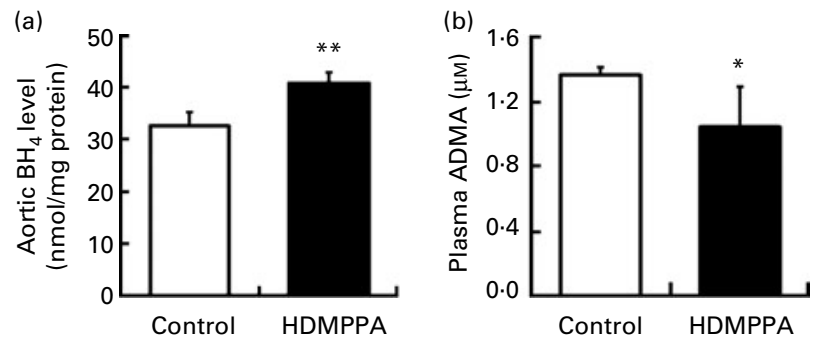

Fig. 2. Effect of $3^{\prime}-\left(4^{\prime}\right.$-hydroxyl-3', $5^{\prime}$-dimethoxyphenyl)propionic acid (HDMPPA) on (a) aortic tetrahydrobiopterin $\left(\mathrm{BH}_{4}\right)$ and (b) plasma asymmetric dimethylarginine (ADMA) in apoE knockout (KO) mice. Control, PBS (vehicle)-treated apoE KO mice; HDMPPA, HDMPPA $10 \mathrm{mg} / \mathrm{kg}$ of body weight-treated apoE KO mice. Values are means, with their standard errors represented by vertical bars $(n 5)$. Mean values were significantly different from those of the control group: ${ }^{\star} P<0.05,{ }^{\star *} P<0.01$. 
$(P<0 \cdot 05$, Fig. 1(c)). These results indicate that HDMPPA inhibited eNOS uncoupling through an increase in NO production and a reduction of ROS in the aorta.

\section{3'-(4'-Hydroxyl-3', 5'-dimethoxyphenyl)propionic acid augmented endothelial nitric oxide synthase activity through increased cofactor and decreased inhibitor}

To see how HDMPPA works on $\mathrm{BH}_{4}$ stabilisation, we measured the biopterin content in the aorta of apoE KO mice (Fig. 2(a)). HDMPPA increased $\mathrm{BH}_{4}$ contents in the aorta of apoE-deficient mice by $25.9 \%$, compared to that of the control group $(P<0.05)$ while, as shown in Fig. 2(b), the plasma level of ADMA in the HDMPPA group was significantly decreased $(1.04 v .1 .36 \mu \mathrm{M}, P<0.05)$, compared to that of control mice.

\section{$3^{\prime}$-(4'-Hydroxyl-3', 5'-dimethoxyphenyl)propionic acid decreased the adhesion molecules in the aorta}

To investigate the anti-inflammatory effect of HDMPPA in the aorta of apoE KO mice, we examined the effect of HDMPPA on protein expression of VCAM-1 and ICAM-1 in the aorta by Western blotting. As shown in Fig. 3, protein levels of VCAM-1 and ICAM-1 in the aorta of the HDMPPA group was significantly reduced compared to the control group $(P<0 \cdot 05)$.

\section{3'-(4'-Hydroxyl-3', 5'-dimethoxyphenyl)propionic acid reduced the pro-inflammatory enzymes in the aorta}

The inhibitory effect of HDMPPA on inflammatory response was examined in terms of determining the protein expressions of COX-2 and iNOS by Western blotting. A marked decrease in COX-2 protein expression was observed in the aorta of apoE KO mice treated with HDMPPA $(P<0 \cdot 01$, Fig. 4). Also, the HDMPPA-treated group showed lower protein level of iNOS than the control group in the aorta of apoE $\mathrm{KO}$ mice $(P<0 \cdot 05$, Fig. 4)
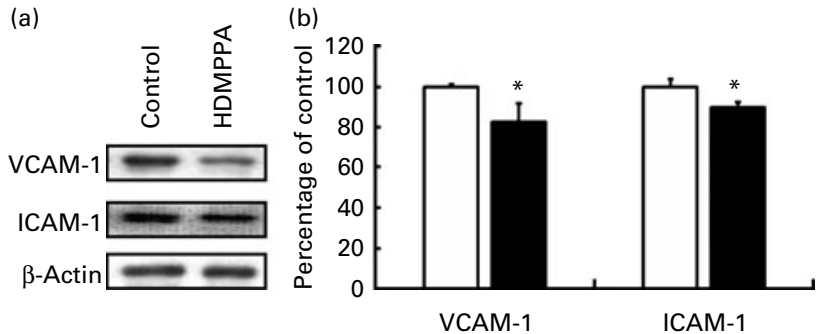

Fig. 3. Effect of $3^{\prime}-\left(4^{\prime}\right.$-hydroxyl-3', $5^{\prime}$-dimethoxyphenyl)propionic acid (HDMPPA) on protein expression of adhesion molecules in the aorta of apoE knockout (KO) mice. Control ( $\square$ ), PBS (vehicle)-treated apoE KO mice; HDMPPA (ם), HDMPPA $10 \mathrm{mg} / \mathrm{kg}$ of body weight-treated apoE KO mice. Values are means, with their standard errors represented by vertical bars $(n 5) .{ }^{*}$ Mean values were significantly different from those of the control group $(P<0.05)$. VCAM-1, vascular cellular adhesion molecule-1; ICAM-1, intercellular adhesion molecule-1.
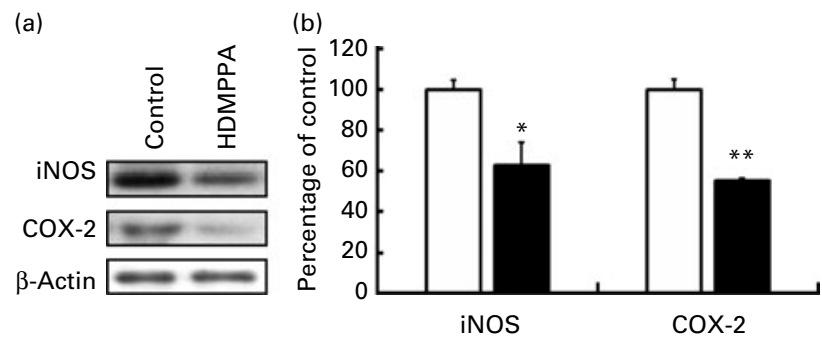

Fig. 4. Effect of $3^{\prime}-\left(4^{\prime}\right.$-hydroxyl-3', $5^{\prime}$-dimethoxyphenyl)propionic acid (HDMPPA) on protein expression of inducible nitric oxide synthase (iNOS) and cyclo-oxygenase-2 (COX-2) in the aorta of apoE knockout (KO) mice. Control ( $\square$ ), PBS (vehicle)-treated apoE KO mice; HDMPPA (ם), HDMPPA $10 \mathrm{mg} / \mathrm{kg}$ of body weight-treated apoE KO mice. Values are means, with their standard errors represented by vertical bars $(n 5)$. Mean values were significantly different from those of the control group: ${ }^{\star} P<0.05,{ }^{\star \star} P<0.01$.

In the HDMPPA group, I $\mathrm{B} \boldsymbol{\mathrm { B }} \alpha$ protein level was significantly increased $(P<0 \cdot 01)$, whereas NF-кB p65 protein level was decreased $(P<0 \cdot 01)$ in the aortic lysate, compared with the control group (Fig. 5).

\section{Discussion}

The alterations of $\mathrm{NO}$ pathway, such as increased NO decomposition by the superoxide anion (formation of peroxynitrite) or altered NO-generating enzyme (mostly eNOS) expression, play a central role in endothelial dysfunction induced by hypercholesterolaemia ${ }^{(24)}$. Under physiological conditions, endothelial stimulation induces the production and release of $\mathrm{NO}$, which diffuses to surrounding tissue and cells and exerts its cardiovascular protective effect by relaxing media-smooth muscle cells, preventing leucocyte adhesion and migration into the arterial wall, muscle cell proliferation, platelet adhesion and aggregation, and adhesion molecule expression $^{(1,2)}$. Several reports have determined that eNOS deficiency accelerates plaque formation, confirming the important role of endothelial NO production for atheroprotection ${ }^{(25,26)}$. Therefore, preventing NO breakdown by ROS and/ or decreased production by eNOS could be a therapeutic target against the process of vascular disease. Moreover, several studies have shown that some agents used to increase $\mathrm{NO}$ production and/or to inhibit the loss of NO bioavailability were helpful to retard the process of vascular disease ${ }^{(27)}$. In this study, HDMPPA treatment significantly augmented NO concentration in the aorta of apoE $\mathrm{KO}$ mice, accompanied by a reduction of ROS levels. In our previous study, we demonstrated that HDMPPA effectively reduced vascular ROS level through inhibiting NADPH oxidase activity in apoE KO mice ${ }^{(18)}$. Furthermore, the protein expression of eNOS was up-regulated in the aorta of the HDMPPA-treated group. These results suggested that HDMPPA could prevent the loss of NO bioavailability via reduction of ROS level and up-regulation of eNOS.

Another important factor related to the preservation of $\mathrm{NO}$ bioactivity is the prevention of eNOS uncoupling, which generates superoxide rather than $\mathrm{NO} . \mathrm{BH}_{4}$ is an essential cofactor required for the activity of eNOS. Recent research demonstrated 
(a)

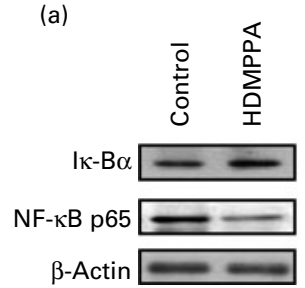

(b)

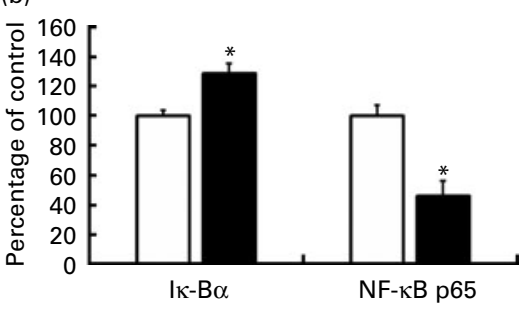

Fig. 5. Effect of $3^{\prime}-\left(4^{\prime}\right.$-hydroxyl-3', $5^{\prime}$-dimethoxyphenyl)propionic acid (HDMPPA) on protein expression of $\mathrm{I}_{\mathrm{K}} \mathrm{B} \alpha$ and NF-KB p65 in the aorta of apoE knockout (KO) mice. Control ( $\square$ ), PBS (vehicle)-treated apoE KO mice;

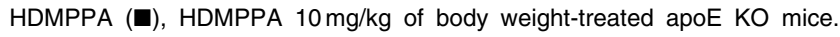
Values are means, with their standard errors represented by vertical bars (n 5). ${ }^{*}$ Mean values were significantly different from those of the control group $(P<0.01)$.

that eNOS activity (to generate NO) can be modestly augmented by increasing $\mathrm{BH}_{4}$ levels even under the normal physiological conditions ${ }^{(28)} \cdot \mathrm{BH}_{4}$ deficiency is believed to lead to eNOS uncoupling, resulting in impaired endothelium-dependent vasodilation and the production of superoxide radicals from the uncoupled enzymatic form. Additionally, an increased oxidation of $\mathrm{BH}_{4}$ is often considered as a mechanism explaining $\mathrm{BH}_{4}$ deficiency in several vascular conditions ${ }^{(29)}$. Laursen et al. ${ }^{(30)}$ demonstrated that peroxynitrite was the principal ROS which oxidises $\mathrm{BH}_{4}$. In this study, HDMPPA showed scavenging activity of peroxynitrite in vitro (data not shown) and inhibited ROS generation in the aorta, suggesting that HDMPPA might further prevent the formation of peroxynitrite. Moreover, the HDMPPA-treated group showed higher $\mathrm{BH}_{4}$ concentration than that of the control group, suggesting that HDMPPA could prevent the oxidation of $\mathrm{BH}_{4}$. Therefore, HDMPPA elevated protein expression of eNOS, at least in part, through increased $\mathrm{BH}_{4}$ levels.

In addition, eNOS activity is determined by an endogenous inhibitor, ADMA, which inhibits cellular L-arginine uptake by endothelial cells, leading to the reduction of NO generation from L-arginine. It is postulated that altered NO bioavailability may result from an increase in ADMA, which may be a critical factor for vascular disease $\mathrm{sin}^{(31-33)}$. Circulating ADMA levels have been assessed in a variety of systemic CVD, and are increased in conditions associated with hypercholesterolaemia, atherosclerosis, hypertension, chronic renal failure and chronic heart failure ${ }^{(34)}$. Animal studies using the rabbit, rat and mouse have shown that the concentration of ADMA in the plasma was increased under a variety of hypercholesterolaemic conditions ${ }^{(35-37)}$. Administration of exogenous ADMA for 4 weeks aggravated atherosclerotic lesions both in apoE $\mathrm{KO}$ mice and in $\mathrm{C} 57 \mathrm{BL} / 6 \mathrm{~J}$ mice ${ }^{(36)}$. Other studies have documented that lysophosphatidylcholine or oxidised-LDL elevated the production of ADMA while certain antioxidants such as vitamin E, probucol and xanthines markedly prevented the elevation of ADMA in the plasma ${ }^{(38,39)}$. Therefore, plasma ADMA may be involved in the progression of atherosclerosis, and therefore, the reduction of ADMA beneficially influences the process of vascular disease. In the present study, HDMPPA significantly decreased plasma ADMA levels in apoE KO mice. These data suggest that HDMPPA improves reduced NO bioactivity and eNOS expression in the aorta of atherosclerotic mice via decreased plasma ADMA.

Atherosclerosis is a chronic inflammatory process which is involved and interacted with various factors such as immunomodulatory compounds, immune cells and blood lipid profile ${ }^{(40)}$. Elevated LDL oxidation by ROS as well as a loss of vascular protective effect of $\mathrm{NO}$ are strongly associated with the inflammatory process of atherosclerosis ${ }^{(41)}$. As an initial event in the pathogenesis of atherosclerosis, expression of adhesion molecules, especially both of ICAM-1 and VCAM-1, plays a central role in the recruitment of circulating monocytes and invasion into the intima. Up-regulation of VCAM-1 and ICAM-1 at the endothelial cell surface initiates pathological leucocyte-endothelial cell interaction, which ultimately exposes the vascular wall and surrounding tissues to the damaging action of activated leucocytes and causes the subsequent development of endothelial dysfunction/atherosclerosis ${ }^{(42-44)}$ COX-2 induces the pathogenesis of inflammatory disorders in response to the production of a variety of inflammatory cytokines, many of which are known to be produced during the progression of atherosclerosis ${ }^{(45)}$. The elevated expression of iNOS has been observed in atherosclerotic lesions where high amounts of NO are produced and combine with superoxide, which might enhance the progression of atherosclerosis $^{(46,47)}$. In addition, the deficiency of iNOS reduced the progression of atherosclerosis ${ }^{(48)}$. Our results showed that HDMPPA treatment markedly reduced the protein expression of VCAM-1 and ICAM-1 in the aorta of apoE KO mice. Moreover, pro-inflammatory iNOS and COX-2 expression in the aorta were decreased by HDMPPA treatment. These results indicate that HDMPPA suppressed the inflammatory responses in the aorta of apoE KO mice.

It has been shown that the activation and translocation of the redox-sensitive transcription factor $\mathrm{NF}-\kappa \mathrm{B}$ are essential for the signal transduction pathway for the expressions of adhesion molecules and pro-inflammatory enzymes ${ }^{(49,50)}$.

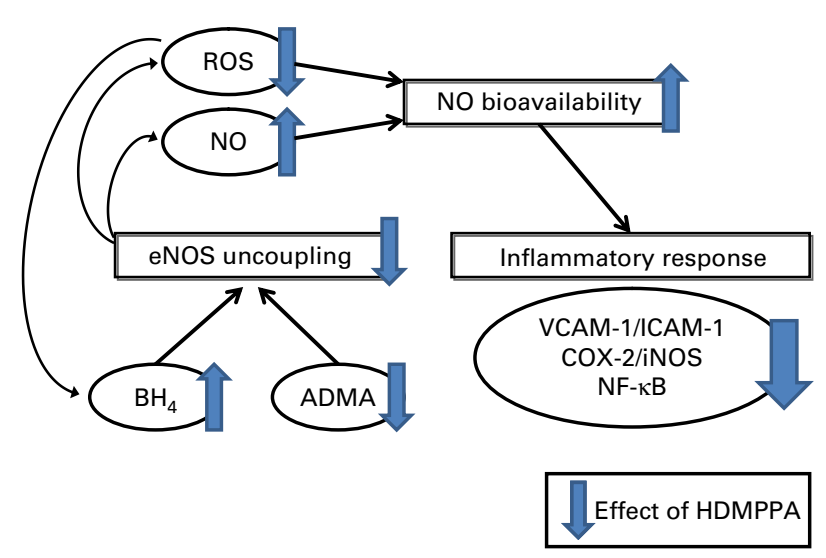

Fig. 6. Overall effects of $3^{\prime}-\left(4^{\prime}\right.$-hydroxyl-3', $5^{\prime}$-dimethoxyphenyl)propionic acid (HDMPPA) on nitric oxide (NO) bioavailability and inflammatory response. ROS, reactive oxygen species; eNOS, endothelial NO synthase; $\mathrm{BH}_{4}$, tetrahydrobiopterin; ADMA, asymmetric dimethylarginine; VCAM-1, vascular cellular adhesion molecule-1; ICAM-1, intercellular adhesion molecule-1; COX-2, cyclo-oxygenase-2; iNOS, inducible NO synthase. (A colour version of this figure can be found online at www.journals.cambridge.org/bjn) 
NF- $\mathrm{B}$ has been implicated as a key mediator of inflammatory response in atherosclerosis ${ }^{(51)}$. This transcription factor is a DNA binding protein complex that is usually present in the cytosol as an inactive complex. ІкB, an associated protein, renders this complex inactive by shielding the nuclear localisation signal. Upon I $\mathrm{KB}$ phosphorylation and its subsequent degradation, the heterodimeric NF- $\mathrm{BB}$ complex translocates from the cytosol to the nucleus, where it binds to specific DNA sequences in the promoter region of several genes and up-regulates their transcription. Most inflammatory genes expressed in endothelial cells during the initial phase of lesion formation and in response to inflammatory mediators are dependent on NF- $\mathrm{KB}$ activation ${ }^{(52)}$. Genes encoding a variety of inflammatory effectors including cytokines, chemokines, growth factors, leucocyte adhesion molecules and inducible enzymes such as iNOS are NF- $\mathrm{BB}$ responsive ${ }^{(53-55)}$. From the results of another report, $\mathrm{H}_{2} \mathrm{O}_{2}$ or oxygen radicals produced during the inflammatory processes act as a second messenger to activate NF-кB directly or indirectly ${ }^{(56)}$. However, numerous studies suggest that ROS inhibitors such as flavonoids, $\alpha$-tocopherol, ascorbate, troglitazone, aspirin, gallate, etc. decrease NF- $\mathrm{KB}$ activation induced by IL- 1 or TNF- $\alpha$ and suppress the activation of adhesion molecules and chemoattractant which are indispensable molecules for atherogenesis ${ }^{(57-61)}$. The present data showed that aortic protein

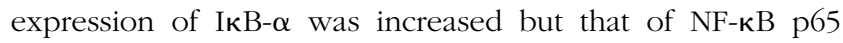
was reduced in the HDMPPA-treated group, compared with the control group. Although we did not examine the nuclear activation of NF- $\mathrm{B}$, our experimental results could demonstrate that HDMPPA regulate inflammatory response by the inhibition of NF- $\mathrm{\kappa B}$ expression, which results may be due to the antioxidant activity of HDMPPA.

Several studies to identify the potential health benefits of kimchi have demonstrated that kimchi exerted its lipid-lowering activity as in experimental animal ${ }^{(10,13-15)}$ and human studies $^{(11,12)}$, and that its active compound, HDMPPA, showed anti-atherosclerotic effect by decreasing the aortic intima thickness and fatty streak size of aortic sinus in hypercholesterolaemic rabbits ${ }^{(16)}$ and mice ${ }^{(18)}$, respectively. On the basis of average kimchi consumption ( $150 \mathrm{~g} / \mathrm{d})$ by Korean adults, the physiological amount of HDMPPA is about $1.5 \mathrm{mg} / \mathrm{d}$. Unfortunately, the dose of HDMPPA used in this study is not physiologically relevant to human consumption levels. However, the pharmacological dose of HDMPPA (for example, pill-format) may be more beneficial for therapeutic applications in human atherosclerosis. In Fig. 6, the anti-atherosclerotic effect of HDMPPA in the aorta of apoE KO mice was depicted. HDMPPA increased NO content in the aorta, while reducing ROS concentration. Furthermore, in the HDMPPA-treated group, aortic eNOS expression was up-regulated compared with the control group. These results suggest that HDMPPA could maintain NO bioavailability through increasing eNOS expression and preventing $\mathrm{NO}$ degradation by ROS. HDMPPA treatment in apoE KO mice inhibited eNOS uncoupling through an increase in vascular $\mathrm{BH}_{4}$ content and a decrease in serum ADMA levels. Moreover, HDMPPA ameliorated inflammatory response in the aorta of apoE KO mice probably through inhibiting NF- $\mathrm{KB}$ expression.
Therefore, the present study suggests that HDMPPA, the active compound of kimchi, a Korean functional food, may exert its vascular protective potential through the preservation of $\mathrm{NO}$ bioavailability and suppression of the inflammatory response.

\section{Acknowledgements}

The present study was supported by a grant (KRF-2005-202F00058) from the Korea Research Foundation funded by the Korean Government. J. S. N. and Y. H. C. conducted the experimental work. Y. O. S. designed the experiment and wrote the manuscript. The authors declare that they have no competing financial interests in relation to this study.

\section{References}

1. Li H \& Forstermann U (2000) Nitric oxide in the pathogenesis of vascular disease. J Pathol 190, 244-254.

2. Li H, Wallerath $\mathrm{T} \&$ Forstermann U (2002) Physiological mechanisms regulating the expression of endothelial-type NO synthase. Nitric Oxide 7, 132-147.

3. Thum T, Fraccarollo D, Schultheiss M, et al. (2007) Endothelial nitric oxide synthase uncoupling impairs endothelial progenitor cell mobilization and function in diabetes. Diabetes 56, 666-674

4. Li H \& Forstermann U (2009) Prevention of atherosclerosis by interference with the vascular nitric oxide system. Curr Pharm Des 15, 3133-3145.

5. Antoniades C, Shirodaria C, Leeson P, et al. (2009) Association of plasma asymmetrical dimethylarginine (ADMA) with elevated vascular superoxide production and endothelial nitric oxide synthase uncoupling: implications for endothelial function in human atherosclerosis. Eur Heart $J$ 30, 1142-1150.

6. Palmer RMJ, Ferrige AG \& Moncada S (1987) Nitric oxide release accounts for the biological activity of endotheliumderived relaxing factor. Nature 327, 524-526.

7. Ross R (1999) Atherosclerosis - an inflammatory disease. $N$ Eng J Med 340, 115-126.

8. Pahl HL (1999) Activators and target genes of Rel/NF-кB transcription factors. Oncogene 18, 6853-6866.

9. Cheigh HS \& Park KY (1995) Biochemical, microbiological and nutritional aspects of kimchi (Korean fermented vegetable products). Crit Rev Food Sci Nutr 34, 175-203.

10. Song YO (2004) The functional properties of Kimchi for the health benefits. Food Ind Nutr 9, 27-33.

11. Kwon MJ, Chun JH, Song YS, et al. (1999) Daily kimchi consumption and its hypolipidemic effect in middle-aged men. J Korean Soc Food Sci Nutr 28, 1144-1150.

12. Choi SH, Kim HJ, Kwon MJ, et al. (2001) The effect of kimchi pill supplementation on plasma lipid concentration in healthy people. J Korean Soc Food Sci Nutr 30, 913-920.

13. Kwon MJ, Song YS, Choi MS, et al. (2003) Red pepper attenuates cholesteryl ester transfer protein activity and atherosclerosis in cholesterol-fed rabbits. Clin Chim Acta 332, 37-44.

14. Kwon MJ, Song YS, Choi MS, et al. (2003) Cholesteryl ester transfer protein activity and atherogenic parameters in rabbits supplemented with cholesterol and garlic powder. Life Sci 72, 2953-2964

15. Kim HJ, Kwon MJ, Seo JM, et al. (2004) The effect of 3-(4'hydroxyl-3', $5^{\prime}$-dimethoxyphenyl)propionic acid in Chinese cabbage kimchi on lowering hypercholesterolemia. J Korean Soc Food Sci Nutr 33, 52-58. 
16. Kim HJ, Lee JS, Chung HY, et al. (2007) 3-(4'-Hydroxyl- $3^{\prime}, 5^{\prime}$ dimethoxyphenyl)propionic acid, an active principle of Kimchi, inhibits development of atherosclerosis in rabbits. J Agric Food Chem 55, 10486-10492.

17. Lee YM, Kwon MJ, Kim JK, et al. (2004) Isolation and identification of active principle in Chinese cabbage kimchi responsible for antioxidant effect. Korean J Food Sci Technol 36, 129-133.

18. Noh JS, Kim HJ, Kwon MJ, et al. (2009) Active principle of kimchi, 3-(4'-hydroxyl-3', $5^{\prime}$-dimethoxyphenyl)propionic acid, retards fatty streak formation at aortic sinus of apolipoprotein E knockout mice. J Med Food 12, 1206-1212.

19. Nishina PM, Verstuyft J \& Paigen B (1990) Synthetic low and high fat diets for the study of atherosclerosis in the mouse. J Lipid Res 31, 859-869.

20. Green LC, Wagner DA, Glogowski J, et al. (1982) Analysis of nitrate, nitrite, and [15N]nitrate in biological fluids. Anal Biochem 126, 131-138.

21. Ali SF, LeBel CP \& Bondy SC (1992) Reactive oxygen species formation as a biomarker of methylmercury and trimethyltin neurotoxicity. Neurotoxicology 13, 637-648.

22. d'Uscio LV \& Katusic ZS (2006) Increased vascular biosynthesis of tetrahydrobiopterin in apolipoprotein E-deficient mice. Am J Physiol Heart Circ Physiol 290, H2466-H2471.

23. Chen BM, Xia LW \& Zhao dRQ (1997) Determination of $N^{\mathrm{G}}$, $N^{\mathrm{G}}$-dimethylarginine in human plasma by high-performance liquid chromatography. J Chromatogr B Biomed Sci Appl 692, 467-471.

24. Harrison DG (1997) Cellular and molecular mechanisms of endothelial cell dysfunction. J Clin Invest 100, 2153-2157.

25. Kuhlencordt PJ, Gyurko R, Han F, et al. (2001) Accelerated atherosclerosis, aortic aneurysm formation, and ischemic heart disease in apolipoprotein $\mathrm{E} /$ endothelial nitric oxide synthase double-knockout mice. Circulation 104, 448-454.

26. Chen J, Kuhlencordt PJ, Astern J, et al. (2001) Hypertension does not account for the accelerated atherosclerosis and development of aneurysms in male apolipoprotein e/endothelial nitric oxide synthase double knockout mice. Circulation 104, 2391-2394.

27. Herman AG \& Moncada S (2005) Therapeutic potential of nitric oxide donors in the prevention and treatment of atherosclerosis. Eur Heart J 26, 1945-1955.

28. Bendall JK, Alp NJ, Warrick N, et al. (2005) Stoichiometric relationships between endothelial tetrahydrobiopterin, endothelial NO synthase (eNOS) activity, and eNOS coupling in vivo: insights from transgenic mice with endothelial-targeted GTP cyclohydrolase 1 and eNOS overexpression. Circ Res 97, 864-871.

29. Moens AL \& Kass DA (2007) Therapeutic potential of tetrahydrobiopterin for treating vascular and cardiac disease. J Cardiovasc Pharmacol 50, 238-246.

30. Laursen JB, Somers M, Kurz S, et al. (2001) Endothelial regulation of vasomotion in apoE-deficient mice: implications for interactions between peroxynitrite and tetrahydrobiopterin. Circulation 103, 1282-1288.

31. Böger RH (2003) Association of asymmetric dimethylarginine and endothelial dysfunction. Clin Chem Lab Med 41, $1467-1472$.

32. Lentz SR, Rodionov RN \& Dayal S (2003) Hyperhomocysteinemia, endothelial dysfunction, and cardiovascular risk: the potential role of ADMA. Atheroscler Suppl 4, 61-65.

33. Cooke JP (2000) Does ADMA cause endothelial dysfunction? Arterioscler Thromb Vasc Biol 20, 2032-2037.

34. Siroen MP, Teerlink T, Nijveldt RJ, et al. (2006) The clinical significance of asymmetric dimethylarginine. Annu Rev Nutr 26, 203-228.
35. Yu XJ, Li YJ \& Xiong Y (1994) Increase of an endogenous inhibitor of nitric oxide synthesis in serum of high cholesterol fed rabbits. Life Sci 54, 753-758.

36. Xiao HB, Yang ZC, Jia SJ, et al. (2007) Effect of asymmetric dimethylarginine on atherogenesis and erythrocyte deformability in apolipoprotein E deficient mice. Life Sci 81, 1-7.

37. Kang KK, Yu JY, Yoo M, et al. (2005) The effect of DA-8159, a novel PDE5 inhibitor, on erectile function in the rat model of hypercholesterolemic erectile dysfunction. Int J Impot Res 17, 409-416.

38. Jiang JL, Li Ns NS, Li YJ, et al. (2002) Probucol preserves endothelial function by reduction of the endogenous nitric oxide synthase inhibitor level. $\mathrm{Br} J$ Pharmacol 135, 1175-1182.

39. Jiang DJ, Hu GY, Jiang JL, et al. (2003) Relationship between protective effect of xanthone on endothelial cells and endogenous nitric oxide synthase inhibitors. Bioorg Med Chem 11, 5171-5177.

40. Lusis AJ (2000) Atherosclerosis. Nature 407, 233-241.

41. Miller YI, Choi SH, Fang L, et al. (2010) Lipoprotein modification and macrophage uptake: role of pathologic cholesterol transport in atherogenesis. Subcell Biochem 51, $229-251$.

42. Davenpeck KL, Gauthier TW, Albertine KH, et al. (1994) Role of $P$-selectin in microvascular leukocyte-endothelial interaction in splanchnic ischemia-reperfusion. Am J Physiol 267, H622-H630.

43. Martin J, Collot-Teixeira S, McGregor L, et al. (2007) The dialogue between endothelial cells and monocytes/ macrophages in vascular syndromes. Curr Pharm Des 13, 1751-1759.

44. Nakashima Y, Raines EW, Plump AS, et al. (1998) Upregulation of VCAM-1 and ICAM-1 at atherosclerosis-prone sites on the endothelium in the ApoE-deficient mouse. Arterioscler Thromb Vasc Biol 18, 842-851.

45. Baker CS, Hall RJ, Evans TJ, et al. (1999) Cyclooxygenase-2 is widely expressed in atherosclerotic lesions affecting native and transplanted human coronary arteries and colocalizes with inducible nitric oxide synthase and nitrotyrosine particularly in macrophages. Arterioscler Thromb Vasc Biol 19, 646-655.

46. Buttery LD, Springall DR, Chester AH, et al. (1996) Inducible nitric oxide synthase is present within human atherosclerotic lesions and promotes the formation and activity of peroxynitrite. Lab Invest 75, 77-85.

47. Luoma JS, Stralin P \& Marklund SL (1998) Expression of extracellular SOD and iNOS in macrophages and smooth muscle cells in human and rabbit atherosclerotic lesions. Arterioscler Thromb Vasc Biol 18, 157-167.

48. Kuhlencordt PJ, Chen J, Han F, et al. (2001) Genetic deficiency of inducible nitric oxide synthase reduces atherosclerosis and lowers plasma lipid peroxides in apolipoprotein E-knockout mice. Circulation 103, 3099-3104.

49. Bu DX, Erl W, de Martin R, et al. (2005) Iк-K $\beta$-dependent NF- $\mathrm{B}$ pathway controls vascular inflammation and intimal hyperplasia. FASEB J 19, 1293-1295.

50. Collins T, Read MA, Neish AS, et al. (1995) Transcriptional regulation of endothelial cell adhesion molecules: NFkappa $\mathrm{B}$ and cytokine-inducible enhancers. FASEB $J \mathbf{9}$ 899-909.

51. Thurberg BL \& Collins T (1998) The nuclear factor-kappa B/ inhibitor of kappa B autoregulatory system and atherosclerosis. Curr Opin Lipidol 9, 387-396.

52. De Martin R, Hoeth M, Hofer-Warbinek R, et al. (2000) The transcription factor NF-kappa B and the regulation of vascular cell function. Arterioscler Thromb Vasc Biol 20, E83-E88. 
53. Lin R, Liu J, Peng N, et al. (2005) Lovastatin reduces nuclear factor kappaB activation induced by C-reactive protein in human vascular endothelial cells. Biol Pharm Bull 28 1630-1634.

54. Baldwin AS (2001) Series introduction: the transcription factor NF-kappaB and human disease. J Clin Invest 107, 3-6.

55. Collins T \& Cybulsky MI (2001) NF-kappaB: pivotal mediator or innocent bystander in atherogenesis? J Clin Invest 107, 255-264.

56. Schreck R, Rieber P \& Baeuerle PA (1991) Reactive oxygen intermediates as apparently widely used messengers in the activation of the NF-kappa B transcription factor and HIV-1. EMBO J 10, 2247-2258.

57. Weber C, Erl W, Pietsch A, et al. (1994) Antioxidants inhibit monocyte adhesion by suppressing nuclear factor-kappa B mobilization and induction of vascular cell adhesion molecule- 1 in endothelial cells stimulated to generate radicals. Arterioscler Thromb 14, 1665-1673.

58. Kharbanda RK, Walton B, Allen M, et al. (2002) Prevention of inflammation-induced endothelial dysfunction: a novel vascular-protective action of aspirin. Circulation 105, 2600-2604.

59. Gerritsen ME, Carley WW, Ranges GE, et al. (1995) Flavonoids inhibit cytokine-induced endothelial cell adhesion protein gene expression. Am J Pathol 147, 278-292.

60. Cominacini L, Garbin U, Pasini AF, et al. (1999) The expression of adhesion molecules on endothelial cells is inhibited by troglitazone through its antioxidant activity. Cell Adhes Commun 7, 223-231.

61. Murase T, Kume N, Hase T, et al. (1999) Gallates inhibit cytokine-induced nuclear translocation of NF-kappaB and expression of leukocyte adhesion molecules in vascular endothelial cells. Arterioscler Thromb Vasc Biol 19, 1412-1420. 